\title{
Sural nerve myelinated fibre density and size in diabetics
}

\author{
J. S. CHOPRA ${ }^{1}$ AND L. J. HURWITZ \\ From the Department of Neurology, Royal Victoria Hospital, Belfast, Northern Ireland
}

Swallow (1966) and O'Sullivan and Swallow (1968) have described the fibre size and content in postmortem specimens of the anterior tibial, sural, and radial nerves from subjects between the ages of 17 and 71 years with no history of peripheral nerve damage. They compared their results with sural nerve biopsies taken from two subjects who had alcoholic and thalidomide neuropathy and concluded that, with a standardized technique, estimation of fibre content and differential fibre size can give useful information in pathological conditions of the peripheral nerves. Chopra (1967a, b) and Chopra, Hurwitz, and Montgomery (1969), by a visual assessment, have reported the pathological features in the sural nerves in patients with diabetes mellitus and with non-diabetic occlusive peripheral vascular disease. In the present communication the result of a study of the myslinated nerve fibre content and differential fibre size of the sural nerves in these patients is presented.

\section{MATERIAL AND METHODS}

Sural nerves from 23 subjects were examined (Table I) constituting seven diabetics without any clinical evidence of peripheral neuropathy (cases $A$ to $G$ ), nine diabetics with objective signs of peripheral neuropathy (cases $\mathbf{H}$ to $P)$, three non-diabetics suffering from chronic occlusive peripheral vascular disease (cases $Q$ to $S$ ), and four controls (cases $\mathrm{T}$ to $\mathrm{W}$ ). All nerves were taken as biopsy specimens except in four controls and two diabetics without neuropathy (cases A and E) which were taken at necropsy. The nerves were all sampled at the level of the lateral malleolus.

Transverse sections, $5 \mu$, from specimens fixed in Fleming's solution were stained with Kultschitsky's haematoxylin, dehydrated and mounted. Details of the patients and the preparation and staining methods have been given previously (Chopra, 1967a; Chopra and Hurwitz, 1967; Chopra et al., 1969).

The fascicles in the transverse section which visually appeared to be technically suitable for fibre counting were noted. Then one of these fascicles which was considered the most representative was selected for fibre

1Present address: Postgraduate Institute of Medical Education and Research, Chandigarh, India. counting. The selected fascicle was photographed with the aid of a Watson optical bench at a magnification of $\times 200$ on Ilford R.U.O. rapid process panchromatic $31 \times 41$ negative plates. For the construction of histograms of fibre size the external diameter of the myelin sheaths was measured from photographs magnified by 1,000 by means of a Perspex cursor and mechanical counter which allowed automatic registration of fibre diameter (Espir and Harding, 1961; Swallow, 1966). The magnification was checked regularly by means of the micrometer before each enlargement. Fibres with an external diameter of $2 \mu$ or less were grouped together and larger fibres were subdivided into $1 \mu$ groups. Fibres of $15 \mu$ or more were also grouped together. The total number of myelinated nerve fibres in each fascicle was also estimated by this method. Fibre density was calculated as the number of fibres per sq. $\mathrm{mm}$ of intraperineural area.

\section{RESULTS}

The fibre density for each patient is given in Table I and illustrated graphically in Figure 1. The mean age of the four controls was 49 years ( 33 to 65 years) and the mean fibre density 5.9 thousand per sq. $\mathrm{mm}$ (range 4.5 to 7.0 thousand per sq. $\mathrm{mm}$ ). The mean age of the diabetics without and with neuropathy was 45.8 and 51.6 years respectively. In all but one of the diabetics with neuropathy (case I, age 38 years) there was considerable loss of myelinated nerve fibres. In the diabetics without neuropathy significant fibre loss was present in three patients (cases B, D and E). In the vascular group the fibre density was within the control range. Figure 2 illustrates the loss of myelinated fibres as seen in transverse sections in diabetic patients compared with a patient with vascular disease and a control.

Histograms of fibre size in each of the 23 cases are shown in Figure 3. There is seen to be a wide variation in fibre size between individual cases. Inspection of the histograms and reference to the fibre density for each nerve (Table I) suggests that there is an absolute loss of large myelinated fibres (11 $\mu$ and over) in eight of the nine cases $(H, I, J, L, M, N, O$, P) of diabetic neuropathy and in two diabetics (E and F) without neuropathy. 
TABLE I

RESULTS OF EXAMINATION OF SURAL NERVES FROM 23 PATIENTS

\begin{tabular}{|c|c|c|c|c|c|c|}
\hline \multirow{2}{*}{$\begin{array}{c}\begin{array}{c}\text { Group } \\
\text { and } \\
\text { case no. }\end{array} \\
\text { Diabetics }\end{array}$} & \multirow[t]{2}{*}{$\begin{array}{l}\text { Age } \\
\text { (yr) }\end{array}$} & \multirow[t]{2}{*}{ Sex } & \multirow[t]{2}{*}{$\begin{array}{l}\text { Fibre density } \\
\text { per sq. mm } \\
\text { (in thousands) }\end{array}$} & $\begin{array}{l}\text { Perc } \\
1-5 \mu\end{array}$ & $\begin{array}{c}\text { entage } \\
6-10 \mu\end{array}$ & $\begin{array}{l}\text { fibres } \\
11 \mu \text { and } \\
\text { over }\end{array}$ \\
\hline & & & & & & \\
\hline A & 27 & $\mathbf{F}$ & 6.0 & $30 \cdot 6$ & $46 \cdot 3$ & $22 \cdot 4$ \\
\hline B & 27 & $\mathbf{M}$ & $3 \cdot 5$ & $40 \cdot 3$ & $34 \cdot 3$ & $25 \cdot 4$ \\
\hline $\mathbf{C}$ & 33 & $\mathbf{F}$ & $5 \cdot 8$ & $27 \cdot 5$ & 50.9 & $21 \cdot 6$ \\
\hline D & 45 & $\mathbf{M}$ & 2.9 & $26 \cdot 8$ & $44 \cdot 4$ & $29 \cdot 8$ \\
\hline $\mathbf{E}$ & 57 & $\mathbf{M}$ & $2 \cdot 6$ & $36 \cdot 2$ & $48 \cdot 2$ & $15 \cdot 6$ \\
\hline $\mathbf{F}$ & 65 & $\mathbf{M}$ & $5 \cdot 7$ & $44 \cdot 0$ & $40 \cdot 2$ & $14 \cdot 6$ \\
\hline $\mathbf{G}$ & 67 & $\mathbf{M}$ & $5 \cdot 5$ & $33 \cdot 5$ & $42 \cdot 9$ & 23.6 \\
\hline Mean & $45 \cdot 8$ & & $4 \cdot 5$ & $34 \cdot 2$ & 43.9 & $21 \cdot 9$ \\
\hline \multicolumn{7}{|c|}{ Diabetics with neuropathy } \\
\hline $\mathbf{H}$ & 29 & $\mathbf{F}$ & $1 \cdot 8$ & $30 \cdot 0$ & $60 \cdot 7$ & $9 \cdot 3$ \\
\hline I & 38 & $\mathbf{F}$ & $5 \cdot 9$ & $25 \cdot 7$ & $58 \cdot 2$ & $16 \cdot 0$ \\
\hline $\mathbf{J}$ & 41 & $\mathbf{F}$ & $1 \cdot 7$ & $23 \cdot 2$ & $43 \cdot 2$ & $33 \cdot 4$ \\
\hline $\mathbf{K}$ & 47 & $\mathbf{F}$ & $3 \cdot 7$ & $17 \cdot 5$ & $46 \cdot 5$ & $35 \cdot 8$ \\
\hline $\mathbf{L}$ & 51 & $\mathbf{F}$ & $1 \cdot 4$ & $15 \cdot 0$ & $73 \cdot 6$ & $12 \cdot 0$ \\
\hline$\overline{\mathbf{M}}$ & 56 & $\mathbf{M}$ & $1 \cdot 5$ & $30 \cdot 0$ & $43 \cdot 3$ & $26 \cdot 6$ \\
\hline $\mathbf{N}$ & 67 & $\mathbf{F}$ & 1.9 & 38.9 & $36 \cdot 5$ & $24 \cdot 5$ \\
\hline $\mathbf{O}$ & 67 & $\mathbf{M}$ & $1 \cdot 3$ & $6 \cdot 2$ & $70 \cdot 2$ & $23 \cdot 2$ \\
\hline $\mathbf{P}$ & 69 & $\mathbf{F}$ & $2 \cdot 7$ & $59 \cdot 7$ & $34 \cdot 7$ & $5 \cdot 5$ \\
\hline Mean & $51 \cdot 6$ & & $2 \cdot 4$ & $27 \cdot 3$ & $51 \cdot 9$ & $20 \cdot 7$ \\
\hline \multicolumn{7}{|l|}{ Vascular } \\
\hline $\mathbf{Q}$ & 41 & $\mathbf{M}$ & $4 \cdot 8$ & $16 \cdot 0$ & $42 \cdot 8$ & $41 \cdot 5$ \\
\hline $\mathbf{R}$ & 57 & $\mathbf{M}$ & $6 \cdot 2$ & $31 \cdot 3$ & $48 \cdot 0$ & $20 \cdot 6$ \\
\hline $\mathbf{S}$ & 64 & $\mathbf{M}$ & $4 \cdot 6$ & $24 \cdot 6$ & $47 \cdot 9$ & $27 \cdot 5$ \\
\hline Mean & $54 \cdot 0$ & & $5 \cdot 2$ & 23.9 & $46 \cdot 2$ & $29 \cdot 8$ \\
\hline \multicolumn{7}{|l|}{ Controls } \\
\hline $\mathbf{T}$ & 33 & $\mathbf{M}$ & $7 \cdot 0$ & $23 \cdot 9$ & $50 \cdot 1$ & $25 \cdot 9$ \\
\hline $\mathbf{U}$ & 44 & $\mathbf{M}$ & $6 \cdot 3$ & $36 \cdot 7$ & $44 \cdot 1$ & $19 \cdot 1$ \\
\hline $\mathbf{V}$ & 54 & $\mathbf{M}$ & $4 \cdot 6$ & $26 \cdot 3$ & $49 \cdot 1$ & $24 \cdot 6$ \\
\hline $\mathbf{w}$ & 65 & $\mathbf{F}$ & 58 & $30 \cdot 6$ & $48 \cdot 5$ & $20 \cdot 9$ \\
\hline Mean & $49 \cdot 0$ & & 5.9 & $29 \cdot 3$ & $47 \cdot 9$ & $22 \cdot 9$ \\
\hline
\end{tabular}

The percentage of small ( 1 to $5 \mu$ ), medium (6 to $10 \mu$ ), and large (11 $\mu$ and over) myelinated fibres and also the mean percentage of each fibre size division for the different patient groups are given in Table I. Histograms were prepared to illustrate the relative proportion of these three divisions of fibre size (Fig. 4). The proportion of small and large fibres varied in controls ( $T$ to $W$ ) but in general each of these two fibre size divisions constituted about one-third to one-half the number of the medium size fibres, very approximately in a 1:2:1 ratio. The large fibres did not make up the most numerous division in any of the control or patient specimens. Except in two patients without neuropathy (B and F) and in two patients with neuropathy ( $N$ and $P$ ), the fibres in the medium range were the most numerous. In patients $J, K$, and $M$ the pattern was similar to that observed in controls, but in the other diabetics with neuropathy it was disturbed. In patient $O$ there was a greater loss

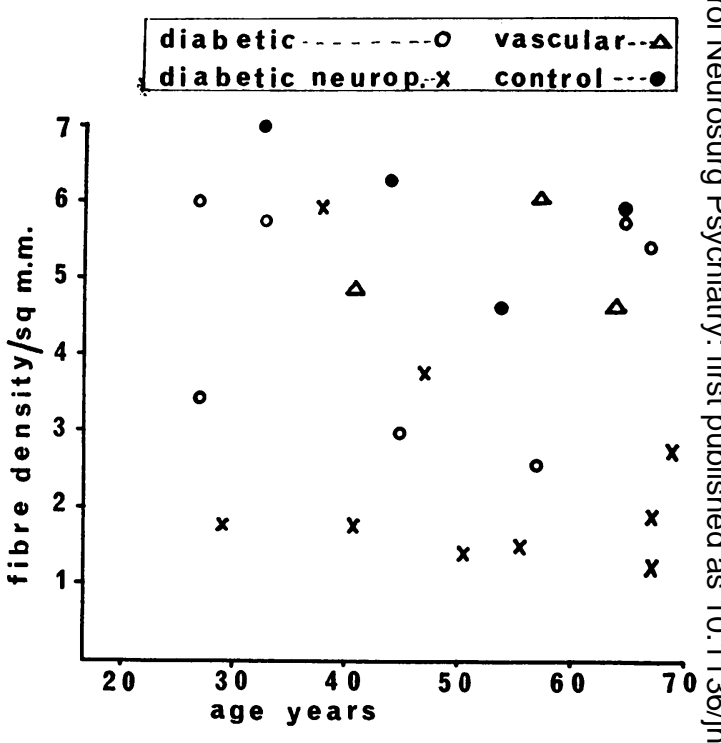

FIG. 1. Nerve fibre density per sq. $\mathrm{mm}$ in patients and controls. $\mathrm{O}=$ diabetic. $\mathrm{x}=$ diabetic neuropathy. $\Delta=\omega$ vascular. $=$ control.

of small size fibres and these represented only $6 \cdot 2$ of the total myelinated nerve fibres. Fibres in the middle range were proportionately much greater i patients $H, I, L$, and $O$, while in patient $P$ the $\frac{D}{O}$ dominant fibres were of the small size range. Cases $\mathbf{H}, \mathbf{L}$, and $\mathbf{P}$ the large myelinated fibres. $\overrightarrow{0}$ represented $12 \%$ or less of the total. In two diabetics of without neuropathy ( $E$ and $F$ ) the large fibres were less than $16 \%$ of the total. The other diabetics and the patients with vascular disease had a pattern similar to that in the controls.

It can be seen that, although there was no constancy in the variations in fibre size from patient to patient, the $1: 2: 1$ ratio for fibres in the small, medium, and large range found in controls was often lost in diabetics and there was also more obvious loss of the large myelinated fibres in diabetics with neuropathy.

\section{DISCUSSION}

Swallow (1966) discussed in detail the merits and demerits of the fixing and staining methods employed by different authors in the past. In his opinion, shrinkage was minimal with the method he employed and which is the one adopted in the present study. This was also established by Duncan (1934), Rexed (1944), and Sanders (1948) who used the osmiumcontaining fluids for fixation. The fibre density in the four controls in this series falls well within the normal range for the sural nerve described by Dyck, 


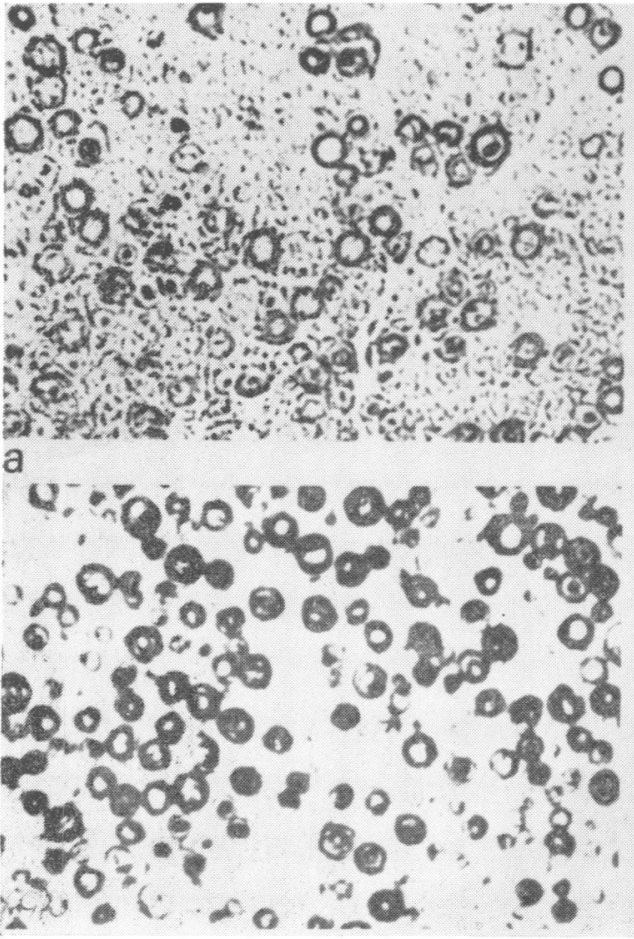

C

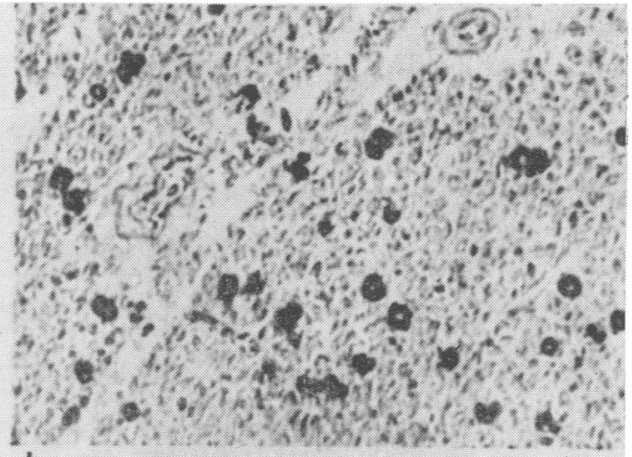

b

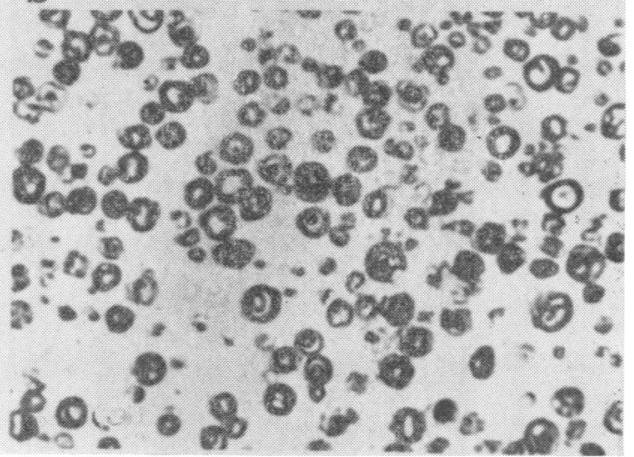

d

FIG. 2. Transverse sections from the sural nerves in four subjects: (a) diabetic without neuropathy (patient D) showing moderate loss of myelinated fibres; (b) diabetic with neuropathy showing severe loss of myelinated fibres (patient H); (c) patient with occlusive vascular disease (patient $Q$ ) with no loss of myelinated fibres; (d) control (patient $T$ ). Kultschitsky's haematoxylin (Van Gieson $\times 200)$.

Beahrs, and Miller (1967), Dyck and Lambert (1966), and O'Sullivan and Swallow (1968).

Although the type of fibre loss varies in all the pathological nerves, the large myelinated fibres were relatively more affected in the diabetics with neuropathy. However, there were more patients in this group (diabetics with neuropathy) who were over 55 years of age, and it has been observed previously that with increasing age there is loss of myelinated fibres (Rexed, 1944; Swallow, 1966; Garven, Gairns, and Smith, 1962). Even making an allowance for the age, there was more loss of myelinated fibres in such patients as compared with the controls of the particular age group. O'Sullivan and Swallow (1968) found a significant selective loss of large myelinated fibres in the elderly. This might have had some influence on the results in the present series among the diabetics with neuropathy, but in three of the younger patients in this group (aged 29, 38, and 51 years; Cases $\mathrm{H}, \mathrm{I}$, and $\mathrm{L}$ ) the loss of large myelinated fibres was greater than in the oldest control nerve and in one of the diabetics with neuropathy (Case $\mathbf{O}$ ) there was a great loss of small myelinated nerve fibres.

In four patients with diabetic neuropathy the fibres in the middle range were present in a relatively much higher percentage than that found in contro nerves. There could be two possible explanationsfirstly, that the small and large myelinated fibres are more affected in the diabetics and the medium size fibres can withstand damage relatively better. The second reason could be that this fibre size division also contained those large myelinated fibres which have either regenerated or remyelinated after degeneration and demyelination. Segmental demyelination and remyelination is the main pathological change in the peripheral nerves of diabetics (Thomas and Lascelles, 1965, 1966; Chopra, 1967a; Chopra et al., 1969) and it has been observed that regenerated and remyelinated fibres do not regain the original diameter of the undamaged nerve fibre (Lascelles and Thomas, 1966; Chopra, 1967a; Chopra et al., 1969).

As segmental demyelination is the main patho- 

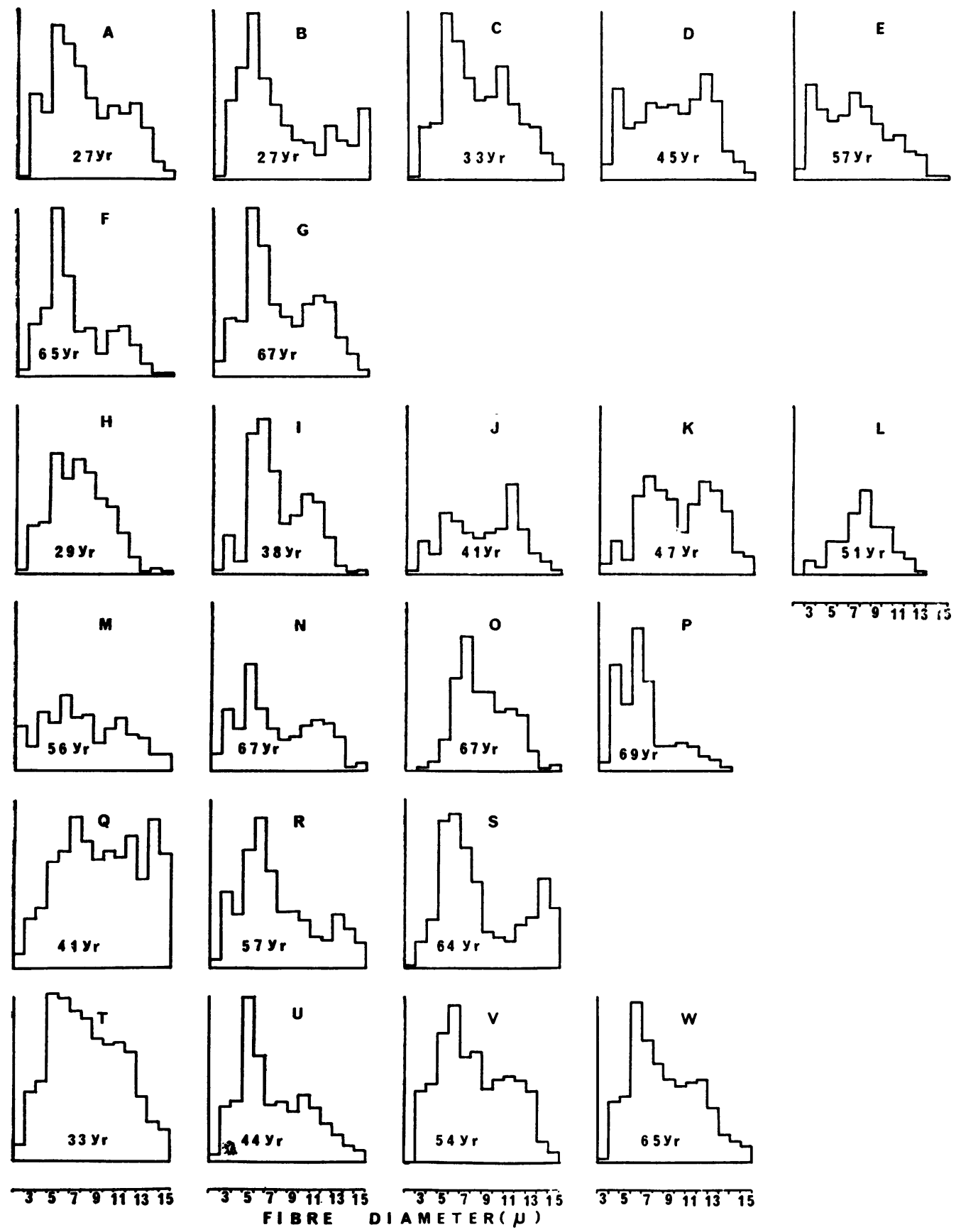

FIG. 3. Histograms showing the distribution of fibre size: $A-G=$ diabetics without neuropathy; $H-P=$ diabetics with neuropathy; $Q-S=$ Patients with occlusive vascular disease; $T-W=$ controls. 

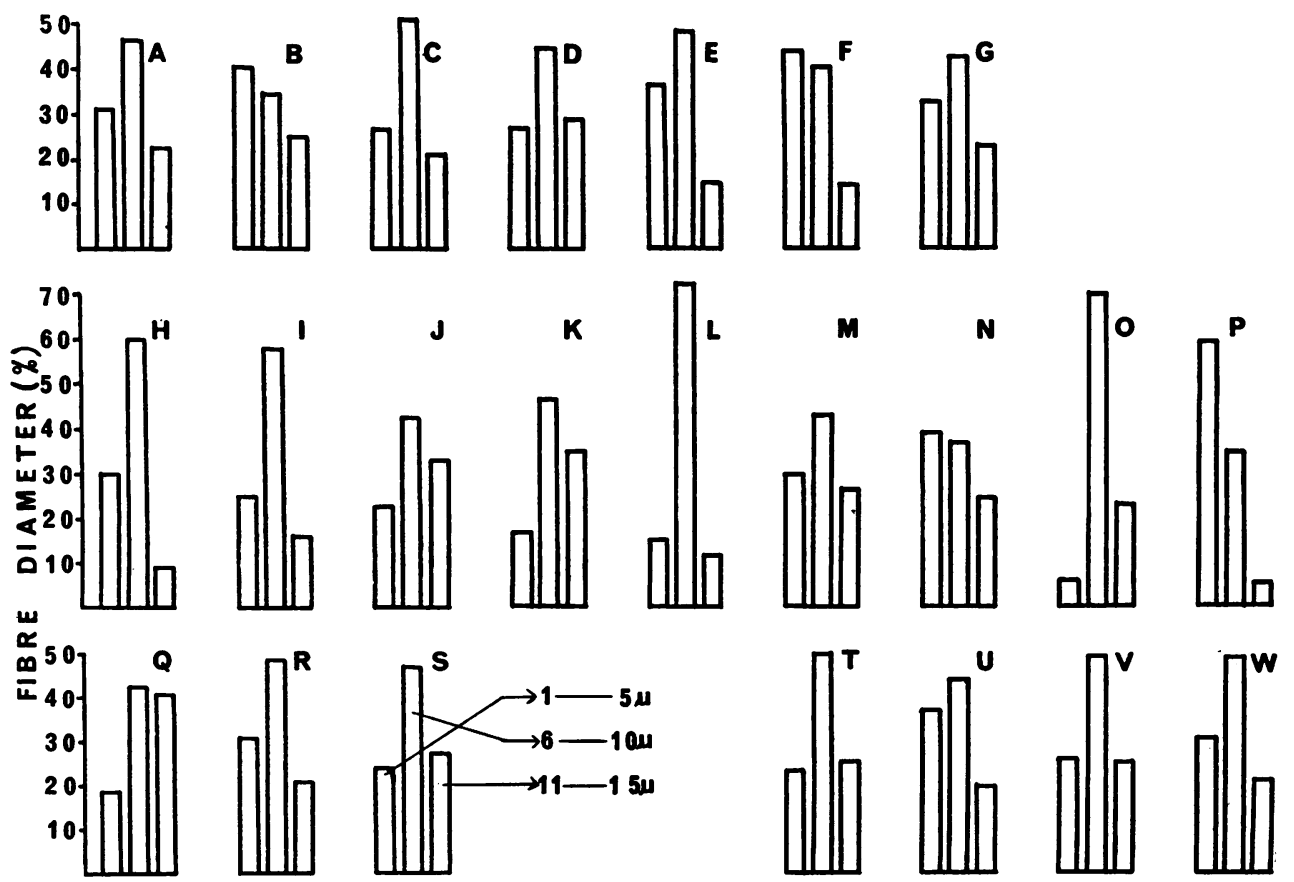

FIG. 4. Histograms showing the relative proportions of small $(1-5 \mu)$, medium $(6-10 \mu)$, and large (11 $\mu$ and over) size nerve fibres in patients and controls. Cases $A-W$ as listed in Figure 3.

logical process in diatetics the validity of the fibre counts in the present study must be questioned as the counting of the fibres was made at one transverse level only. Therefore this may not represent a true fibre content of the rest of the rerve and serial sections would perhaps give more reliable results. Nevertheless, fibre content was assessed visually at different levels in these nerves at a distance of one centimetre and it was considered that the pattern of fibre size and content was approximately the same.

Garven et al. (1962) described a considerable loss of myelinated fibres and selective loss of large myelinated fibres in patients suffering from occlusive arterial disease. This finding was not observed in the three cases in the present series. However, the patients described by Garven, Gairns, and Smith had very severe occlusive vascular disease and a greater loss of myelinated fibres is to be expected in such cases (Eames and Lange, 1967).

This study also confirms earlier suggestions (Chopra, 1967a, b; Chopra et al., 1969) that the pathological changes in diabetic neuropathy are mainly due to a metabolic disturbance. Diabetic patients with good peripheral pulsations and patent vasa nervorum (Chopra et al., 1969) have shown more loss of myelinated fibres than patients suffering from occlusive peripheral vascular disease. Such a loss of large myelinated fibres would explain the signs of neuropathy and reduction in the speed of nerve conduction (Thomas, 1960; Garven et al., 1962; Thomas and Fullerton, 1963).

This study would confirm the view of O'Sullivan and Swallow (1968) that peripheral nerve fibre content and fibre size can be a useful additional investigation in the study of peripheral neuropathies of varying aetiology.

\section{SUMMARY}

Fibre size and density of the myelinated nerve fibres of the sural nerve were examined in 16 diabetic patients, nine of whom had objective evidence of peripheral neuropathy. The results are compared with non-diabetic patients suffering from chronic occlusive peripheral vascular disease and controls. Loss of myelinated nerve fibres of all sizes and a deviation, from controls, in the relative proportion of small, medium, and large fibres was observed only in the diabetic patients and especially in patients with neuropathy. Loss of large myelinated fibres was severe in some of the diabetics with neuropathy.

We are indebted to Dr. M. Swallow for his advice in the present study and for helpful criticism, and grateful to 
Dr. Ingrid Allen for the help and co-operation received from the Department of Pathology, the Queen's University of Belfast. We wish to thank Mr. D. Mehaffey and Mr. R. G. Wood for the microphotography and magnified photo prints; and Miss $M$. Weller for the secretarial help. A generous grant from the Medical Research Council is acknowledged.

\section{REFERENCES}

Chopra, J. S. (1967a). An Electromyographic Study of the Neuromuscular Complications of Diabetes Mellitus. Ph.D. Thesis, Queen's University of Belfast.

- (1967b). Diabetic neuropathy. A histopathological study of the sural nerve. Sixth Congr. Internat. Diabetes Fed., Excerpta medica (Amst.) Int. Congr. Ser., No. 140, p. 5.

fibres in chronic occlusive vascular disease. J. Neurol Neurosurg. Psychiat., 30, 207-214.

- Hurwitz, L. J., and Montgomery, D. A. D. (1969). The pathogenesis of sural nerve changes in diabetes mellitus. Brain, in press.

Duncan, D. (1934). A relation between axone diameter and myelination determined by measurement of myelinated spinal root fibres. J. comp. Neurol., 60, 437-472.

Dyck, P. J., Beahrs, O. H., and Miller, R. H. (1967). Peripheral nerves in hereditary neural atrophies: number and diameter of myelinated fibres. Pp. 673-677 in Recent Advances in Clinical Neurophysiology. Edited by L. Widen and others. Elsevier, Amsterdam.

_- and Lambert, E. H. (1966). Numbers and diameters of nerve fibres and compound action potential of sural nerve; controls and hereditary neuromuscular disorders. Trans. Amer. neurot. Ass., 91, 214-217.

Eames, R. A., and Lange, L. S. (1967). Clinical and pathologica政 study of ischaemic neuropathy.J. Neurol. Neurosurg. Psychiat. 30, 215-226.

Espir, M. L. E., and Harding, D. T. C. (1961). Apparatus for measur ing and counting myelinated nerve fibres. $J$. Neurol. Neurosurg Psychiat., 24, 287-290.

Garven, H. S. D., Gairns, F. W., and Smith, G. (1962). The nervo fibre populations of the nerves of the leg in chronic occlusive arterial disease in man. Scot. med. J., 7, 250-265.

Lascelles, R. G., and Thomas, P. K. (1966). Changes due to age in internodal length in the sural nerve in man. J. Neurol. Neuro. surg. Psychiat., 29, 40-44.

O'Sullivan, D. J., and Swallow, M. (1968). The fibre size and content? of the radial and sural nerves. J. Neurol. Neurosurg. Psychiat 31, 464-470.

Rexed, B. (1944). Contributions to the knowledge of the postnata development of the peripheral nervous system in man. Acto psychiat. (Kbh.), Suppl. 33, 1-206.

Sanders, F. K. (1948). The thickness of the myelin sheaths of norma and regenerating peripheral nerve fibres. Proc. roy. Soc. B. ॄ్ 135, 323-357.

Swallow, M. (1966). Fibre size and content of the anterior tibial $\vec{b}$ nerve of the foot. J. Neurol. Neurosurg. Psychiat., 29, 205-213:-

Thomas, P. K. (1960). Motor nerve conduction in the carpal tunnelsyndrome. Neurology (Minneap.), 10, 1045-1050.

, and Fullerton, P. M. (1963). Nerve fibre size in the carpal tunnet syndrome. J. Neurol. Neurosurg. Psychiat., 26, 520-527.

- and Lascelles, R. G. (1965). Schwann-cell abnormalities in diabetic neuropathy. Lancet, 1, 1355-1357.

- (1966). The pathology of diabetic neuropathy. Quart.J. Med., 489-509. 